\title{
DIE PLANUNG IN DER WIRTSCHAFTSVERFASSUNG DER LATEINAMERIKANISCHEN STAATEN
}

I.

Die Wirtschaftspolitik, besser noch die gesamte Entwicklungspolitik in den iberoamerikanischen Ländern hat seit Beginn der 60er Jahre dadurch eine neue Ausrichtung und systematischere Grundlage erhalten, daß man sich jetzt immer ernsthafter mit der Notwendigkeit der Planung befaßt. Planung beinhaltet vorausschauende Festlegung von in der Zukunft zu erreichenden Zielen auf Grund sorgfältiger Berechnung der vorhandenen Mittel und des einzuschlagenden Weges. Planung unterscheidet sich von allgemeiner Wirtschaftspolitik dadurch, daß für die Zukunft eine Lenkung, mindestens aber eine Orientierung aller wirtschaftlichen und sozialen Vorgänge festgelegt wird. Die bisherigen Maßnahmen der Wirtschaftspolitik der iberoamerikanischen Länder, wie die häufigen Interventionen auf den Gebieten der Devisen- und Importbewirtschaftung, können nicht als „Planung" angesehen werden ${ }^{1}$. Sie waren oft aus der Not geborene Eingriffe, um einem Verfall der Wirtschaft Einhalt zu gebieten. Auch z. B. der Vertrag von Montevideo von 1960 zur Errichtung einer Freihandelszone (ALALC) ist kein „Plan“ im eigentlichen Sinne, da nur das Wunschziel einer in 12 Jahren zu erreichenden Freihandelszone festgelegt wird, ohne die Grundlage einer rationalen Erfassung von Mitteln und Wegen.

Den eigentlichen Anstoß, die „planificación“ der gesamten Entwicklung als Aufgabe zu sehen, gab die "Deklaration von Punta del Este" von 1961. Hierin haben sich die lateinamerikanischen Länder verpflichtet, ,in Ubereinstimmung mit den Grundsätzen der Demokratie weitreichende und gut angelegte wirtschaftliche und soziale Entwicklungsprogramme durchzuführen zwecks Erreichung der wirtschaftlichen Unabhängigkeit " ${ }^{\text {. }}$. Unter der Voraussetzung eines Höchstmaßes eigener Anstrengungen und des Einsatzes nationaler Mittel sollen zusätzlich die iberoamerikanischen Länder ausreichende Finanzhilfen aus dem Ausland - vor allem von den USA - erhalten, und zwar für die nächsten zehn Jahre insgesamt mindestens 20 Milliarden US-Dollar aus allen ausländischen Quellen. Die iberoamerikanischen Länder sind dieser Verpflichtung zur Aufstellung von längerfristigen Plänen weitgehend nachgekommen, zumal auch andere Instiutionen wie die Weltbank auf systematischere Planung drängen.

Vor 1961 hatte seit etwa 1949 die UN-Wirtschaftskommission für Lateinamerika (CEPAL) eine nicht wegzudenkende Vorarbeit geleistet. Bereits 1953 hatte die CEPAL z. B. eine Broschüre herausgegeben mit dem Titel: „An Introduction to the Technique of Programming"'. Insgesamt hat sich allerdings die Arbeit der CEPAL erst nach 1961 in Zusammenhang mit dem praktischen Anstoß durch die Deklaration von Punta del Este voll auswirken können. Behrendt hat festgestellt, daß trotz etwa dreizehnjähriger eifriger Tätigkeit der CEPAL keines der 19 Länder Iberoamerikas 1961 brauchbare Pläne aufzuweisen hatte ${ }^{3}-$ jedenfalls nicht im Sinne einer globalen Entwicklungsstrategie. - Die Grundsätze für eine iberoamerikanische Entwicklungsplanung werden heute in dem "Instituto LatinoAmericano de Planificación Económico y Social" (ILPES) herausgearbeitet, welches 1962 von der CEPAL mit dem Sitz in Santiago de Chile errichtet wurde. In diesem Institut arbeitet eine Gruppe von Volkswirten und Soziologen; den Regierungen soll auf Anforderung die nötige Hilfe bei der Programmierung geboten werden.

\footnotetext{
1 Hierzu: Raúl Prebisch, Wirtschaftsplanung und Demokratie in Lateinamerika, S. 166, in: „Lateinamerika“, Eichholz-Verlag, Bonn 1965. Ulrich Scheuner, Verfassungsrechtliche Probleme einer zentralen staatlichen Planung, S. 75, in: Planung I, Nomos Verlag, Baden-Baden 1965.

2 Carta von Punta del Este, II. Abschnitt, Kap. 1, Ziff. 1.

3 Richard F. Behrendt, Soziale Strategie für Entwicklungsländer, S. 387, S. Fischer Verlag, 1965
} 
Die Einsicht, daß Wirtschaftsplanung in Iberoamerika heute umfassende Entwicklungsplanung sein muß, liegt auch der Arbeit der CEPAL zu Grunde, wenn bei ihr auch die Planung der industriellen Entwicklung ein Hauptarbeitsziel ist. Die Faktoren sozialen und politischen Charakters dürfen nicht übersehen werden, da sie die Lösung der wirtschaftlichen Probleme stark beeinflussen ${ }^{4}$. Neben den drängenden wirtschaftlichen Problemen werden Probleme wie Hebung des Lebensstandards, Integrierung aller Volksschichten, soziale Gleichstellung und bessere Einkommensverteilung ausdrücklich genannt. Auch im Rahmen der Carta von Punta del Este wurden weitere Pläne auf den Gebieten der Erziehung, des Gesundheitswesens und der Steuerreform beschlossen. Insgesamt muß man also von einer Art umfassender interdisziplinärer Gesamtplanung sprechen.

Die sich herauskristallisierende Wirtschaftsauffassung geht aus von der Erkenntnis, daß die Zeit des wirtschaftlichen Liberalismus vorüber und daß eine systematische Behandlung der Entwicklung unerläßlich sei. "The free play of these forces has failed to bring about well-balanced technical penetration into all productive activities in the peripheral countries." 5 Das freie Spiel der Kräfte habe keine Ordnung bewirkt, die Auffassung "laisser faire" sei nur möglich in "argumentos de opereta " und niemals im wirklichen Leben ${ }^{6}$. Es bildet sich eine Einstellung zur Wirtschaft heraus, die sich von der klassisch liberalen Idee eines unbeschränkten privaten Wettbewerbes eindeutig trennt - die sich aber ebenso von der totalitären Planwirtschaft absetzt. Die Erhaltung und Förderung der privaten Initiative und ihre Einbeziehung in die Gesamtplanung als „elemento orgánico integrante" als unerläßlich betrachtet. Die private Initiative müsse zwar orientiert und auch kontrolliert werden, doch hänge von ihr der größte Teil des Erfolges ab8. Derartige Äußerungen dürften heute als symptomatisch anzusehen sein für die sich als geltend durchsetzende Wirtschaftsauffassung. „Wirtschaftsplanung und Integration sind von wachsender Bedeutung im Denken der lateinamerikanischen Volkswirtschaften nach dem Kriege. Ihre theoretischen Grundlagen haben sich nach und nach herausgebildet und sind heute Allgemeingut der Volkswirte, die an der Entwicklung Lateinamerikas interessiert sind ... Über die Grundsätze besteht heute Einmütigkeit." $\theta$

Man ist sich ferner darüber einig, daß dem heutigen Staat eine Hauptrolle bei der Planung zukommt, da er Zielsetzung, Formulierung und staatliche Intervention zu entscheiden habe ${ }^{10}$. Die Rolle des Staates wird in den Betrachtungen der iberoamerikanischen Ơkonomen weniger theoretisch, sondern mehr von der praktischen Seite her begründet. In einer modernen Wirtschaft müsse der Staat Einnahmen haben, er müsse teilhaben an internationalen Beziehungen und er müsse die monetäre Gesetzgebung erlassen. Er beeinflusse die Preise und die Verteilung der Investitionsmittel und damit die Struktur der Produktion und des Wachstums ${ }^{\mathbf{1 1}}$. Die Iberoamerikaner erweisen sich als weniger beschwert mit ideologischen Auseinandersetzungen im Vergleich $\mathrm{zu}$ dem traditionsbelasteten Europa. Ein ideologisches "Tabu“ allein in bezug auf das Wort „Planung", wie in der BRD in den fünfziger Jahren, wäre in Iberoamerika kaum denkbar. Die Entwicklungsprobleme sind so groß und dringend, daß sie eine rasche pragmatische Lösung erfordern.

\footnotetext{
4 Victor L. Urquidi, Posibilidades de una Programación efectiva del Crecimiento en America Latina, S. 19. In: Revista de Economía Latino-Americana, Nr. 3, 1961, Caracas, Juli/September 1961, Publicación bajo los Auspicios del Banco Central de Venezuela.

5 Economic Development, Planning and International Cooperation, CEPAL Schrift E/CN. 12/582/Rev. 1., Santiago 1961, S. 44

6 Jorge Ahumada, Teoría y Programación del Desarrollo Económico, S. 48, Santiago de Chile 1967, Cuadernos del Instituto de Planificación Ec. y. Soc., Nr. 1.

7 Jose A. Guerra y Enrique Lerdau, Planeación e Integración en America Central, in: Economía Latinoamericana, Vol. I, Nr. 3, Juli 1964 (Hrsg. Pan Am. Union), S. 352.

8 Hector Soza Valderrama, Planificación del Desarrollo Industrial, 1. Aufl., 1966, Siglo XXI Editores S. A., Mexico D. F.

9 Guerra-Lerdau, a. a. O., S. 351 f.

10 Hierzu auch: Prebisch, a. a. O., S. 167, 171.

11 Ahumada, a. a. O., S. 48.
} 
II.

Die in den Verfassu nge n niedergelegten Auffassungen vom Verhältnis des Staates zur Wirtschaft haben sich im Laufe der Jahre gewandelt. Die älteren Verfassungen gehen von der klassisch liberalen Doktrin aus und gewähren dem Individuum uneingeschränkten Schutz des privaten Eigentums und der Gewerbefreiheit neben dem außerdem üblichen Katalog von Grundrechten. Danach steht dem Staat keinerlei Recht zur Planung des wirtschaftlichen Ablaufes zu, weder im Sinne einer orientierenden planenden Einwirkung noch gar im Sinne einer alle Kräfte und Mittel verplanenden Zwangswirtschaft. Tatsächlich haben sich die iberoamerikanischen Staaten gegenüber dem eigentlichen Ablauf der Wirtschaft weitgehend indolent gezeigt, was sich bis in unsere Tage auswirkt. Wenn Hirschman ${ }^{12}$ 1957 feststellt, daß es gegenüber der Planung noch keinen "whole-hearted official support" gäbe, so liegt die Wurzel dieses Verhaltens sicherlich auch in dem Fortwirken liberalen Gedankengutes. Das Gleiche wird man auch bezüglich der immer wieder beklagten Passivität der offiziellen Stellen gegenüber den Bestrebungen der ALALC zur Errichtung der Lateinamerikanischen Freihandelszone sagen können.

In den neueren Verfassungstexten finden sich allerdings immer stärker werdende Niederschläge moderner Auffassungen. Die Grundrechte wie Eigentum und Gewerbefreiheit werden zwar weiterhin garantiert, jedoch nur insoweit, als ihre Ausübung im Einklang mit den sozialen Verpflichtungen steht ${ }^{13}$. So heißt es in Artikel 47 der Verfassung von Ekuador von 1967: „Der Staat erkennt an und garantiert das Recht auf privates Eigentum, insoweit es die ihm innewohnende soziale Funktion erfüllt." Ahnlich lautet Artikel 7 Ziffer i) der Verfassung von Bolivien von 1967 (auch Artikel 132).

In beiden genannten Verfassungen finden sich sogar ausdrückliche Verpflichtungen des Staates zur Planung. Die Verfassung von Ekuador enthält in den Artikeln 94/96 einen Abschnitt „De la Planificación“, und es heißt in Artikel 94:

„Der Staat verpflichtet sich, über die wirkungsvolle Nutzung der nationalen Werte $\mathrm{zu}$ wachen und die angeordnete und unterhaltene Entwicklung der Wirtschaft $\mathrm{zu}$ fördern. In Verfolg dessen wird er seine Tätigkeit einem langfristigen Plan unterwerfen, welcher die geeigneten Mittel umfaßt, mit denen unter Anteilnahme aller Einwohner die konkreten Ziele der wirtschaftlichen Entwicklung und des sozialen Fortschrittes erreicht werden sollen."

In Artikel 96 heißt es dann:

„Diese Pläne ... haben verpflichtenden Charakter für den öffentlichen Sektor und indikativen und orientierenden für den privaten."

In ähnlicher Weise wird auch in der Verfassung Boliviens die Pflicht des Staates zur Programmierung statuiert (Artikel 144 I). Die private Initiative soll Ermunterung und Kooperation des Staates genießen, wo sie zur Verbesserung der nationalen Wirtschaft beiträgt (Artikel 144 II). In Artikel 141 statuiert der Staat in einer Generalklausel sein Recht, durch Gesetz die Ausübung von Handel und Industrie zu regulieren, sobald öffentliche Sicherheit und Notwendigkeit dies erfordern.

Es ist interessant, festzustellen, daß sich diese neuen Verfassungsbestimmungen in Ubereinstimmung befinden mit der herrschenden Meinung der Volkswirte. Zugleich entsprechen sie demokratischen Grundsätzen, nach denen es darauf ankommt, in einer "indikativen“ (nicht „imperativen“) Planung die individuelle Tätigkeit mit den lenkenden Maßnahmen des Staates zu verbinden ${ }^{14}$.

Sozialrevolutionäre Ziele waren übrigens zum ersten Male in der heute noch geltenden mexikanischen Verfassung von 1917 enthalten, nachdem hier mehr als

\footnotetext{
12 Alberto O. Hirschman, Ideologies of Economic Development in Latin America, S. 164, in: The Dynamics of Change in Latin American Politics, Ed. by John D. Martz, Englewood Cliffs, N. J., 1965.

13 J. Lloyd Mecham, Latin American Constitutions: Nominal an Real, S. 36, in: The Dynamics of Change in Latin American Politics, Edited by John D. Martz, 1965, Englewood Cliffs, N. J.

14 Scheuner, a. a. O., S. 80.
} 
in anderen iberoamerikanischen Ländern das soziale Problem durch das ganze 19. Jahrhundert hindurch Gegenstand heftiger Auseinandersetzungen gewesen war. Die vor der mexikanischen Revolution von 1910 bestehenden Zustände wirtschaftlicher Ausbeutung als Folge eines schrankenlosen "laisser faire, laisser aller" waren im übrigen typisch für ganz Iberoamerika. México hat sich heute, durch ein eigenes System einer realen Mischung von Kapitalismus und Sozialismus, von staatlicher Lenkung und privater Initiative, auf der Basis eines stabilen Herrschaftssystems eigener iberoamerikanischer Prägung, die Möglichkeit langfristiger Planungen geschaffen. Bereits 1933 wurde unter Präsident Calles zum ersten Male ein Wirtschaftsplan (Plan Sexenal) aufgestellt. Diese frühen Pläne sind sicherlich noch keine Entwicklungspläne im heutigen Sinne, sie zeigen aber, welche Voraussetzungen ein Land schaffen muß, um wirkungsvolle Pläne aufstellen wie auch durchführen zu können. México nimmt in Iberoamerika hierin noch absolut eine Sonderstellung ein - die übrigen Länder stehen vor einer großen Zahl schwerwiegender Fragen, welche die Erfüllung von Entwicklungsplänen häufig problematisch erscheinen lassen.

\section{III.}

Es ist kein Geheimnis, daß zwar eine große Zahl von Entwicklungsplänen aufgestellt worden ist, daß aber die Durchführung oft auf Schwierigkeiten stößt und der Erfolg nicht den hochgespannten Erwartungen entspricht. Angesichts der kurzen Zeit der Aufstellung von Plänen, der sicherlich noch unzulänglichen Popularität dieser Ideen und des noch nicht vollkommenen Instrumentariums ist dies nicht verwunderlich. Darüber hinaus sind aber noch mancherlei Voraussetzungen offen, und zwar sowohl für eine realistische Erstellung von Planungen wie auch für die Durchführung. Grundsätzlich ist die Erfüllung von Planungen niemals mit mathematischer Präzision vorauszusagen: In Iberoamerika sind die Faktoren der Ungewißheit dabei größer als in entwickelten Ländern.

Prebisch hat mit Recht betont ${ }^{15}$, daß Iberoamerika eigene Lösungen brauche, wozu in geistiger Unabhängigkeit eigene Möglichkeiten gefunden werden müßten. Für die besonderen Entwicklungssituationen in den iberoamerikanischen Ländern werden eigene Modelle ausgebildet werden müssen - eine kritiklose Übernahme von Modellen etwa aus den USA bzw. der in Industrieländern gemachten Erfahrungen verbietet sich. Die Ausgangssituation in den iberoamerikanischen Entwicklungsländern ist nicht $\mathrm{zu}$ vergleichen mit derjenigen hochgerüsteter Industriestaaten. Iberoamerika steht in dem Teufelskreis der politischen und wirtschaftlichen Unstabilität, dem Nachhinken des wirtschaftlichen Wachstums hinter der explosiven Bevölkerungszunahme, der Unausgeglichenheit der nationalen Integration, den Erfordernissen der Systematisierung der industriellen Entwicklung, hinter der das Wachstum des Agrarsektors nicht zurückbleiben darf - um nur die Hauptprobleme anzudeuten, die eine grundlegend neue Planung verlangen.

Die für die erfolgreiche Durchführung einer rationalen Planung erforderliche Errechnungsgrundlage ist für Iberoamerika noch ein Sorgenkind. Gewiß hat neuerdings die Erarbeitung von brauchbaren Statistiken erhebliche Fortschritte gemacht. Die Notwendigkeit der Verbesserung der Technik auf statistischem Gebiet ist unter anderem immer wieder von der CEPAL hervorgehoben worden. Der Mexikaner Victor L. Urquidi stellte fest, daß es etwa 1950 noch kein ausreichendes statistisches Instrumentarium gegeben habe, daß dieses auch heute noch unvollkommen sei; man sei aber heute bereits imstande, eine Entwicklung zu programmieren ${ }^{16}$. Diese Bemerkung trifft für México sicherlich $\mathrm{zu}$, für manche anderen Länder dürfte sie zu optimistisch sein.

Ebenso muß es notwendigerweise zunächst an ausgebildeten Fachkräften fehlen, wie auch an organisatorischen Voraussetzungen. In dieser Hinsicht sind nationale Planungsinstitute zu erwähnen, wie z. B. in Perú das „Instituto Nacional de

15 A. a. O., S. $171 \mathrm{f}$.

16 Victor L. Urquidi, a. a. O., S. $21 \mathrm{ff}$. 
Planificación", ein offizielles zentrales Planungsorgan, dessen Direktor Ministerrang besitzt. Seit 1963 wurde eine beachtliche Reihe von Arbeiten über Planung und Probleme der Entwicklungspolitik veröffentlicht. - In der neuen Verfassung von Ekuador von 1967 ist eine "Junta Nacional de Planificación y Coordinación" institutionalisiert (Artikel 235 f.). Derartige Beispiele überall einsetzender ernsthafter Bemühungen ließen sich heute zahlreich anführen.

Ausgesprochene Erschwerungen in der Durchführungen von Planungen liegen in Imponderabilien, die sich aus der iberoamerikanischen Mentalität ergeben. In praktischer Hinsicht steht das seit Jahrhunderten eingebürgerte individualistische Verhalten gegenüber öffentlichen Anforderungen, wie etwa im Bereich des Steuerwesens oder der Kapitalflucht, hindernd im Wege.

In besonderem Maße ist der Iberoamerikaner einem Wunschdenken ausgesetzt, was durch die Carta von Punta del Este und die Aussicht auf hohe Finanzhilfen aus dem Auslande sicherlich noch gefördert worden ist. Auf die Verwandtschaft von Planung und Utopie hat Kaiser hingewiesen ${ }^{17}$; bei dem Iberoamerikaner ist die Neigung zu utopischen Vorstellungen ausgeprägt, wie überhaupt das Emotionelle überwiegt ${ }^{18}$.

Dem Iberoamerikaner liegt bisher mehr das Improvisieren und das Handeln aus dem Augenblick als rationales langfristiges Planen, das der bisherigen traditionellen Mentalität nahezu wesensfremd ist. Die Ansicht, daß Wirtschaften in sich bereits „Planung“ bedeutet, trifft für Iberoamerika nur bedingt zu. Die Art des Wirtschaftens wird bisher im wesentlichen charakterisiert durch das „Hacienda“Denken: die Wirtschaft ist diejenige eines großen autarken Gutsbetriebes' ${ }^{19}$. Man half sich lokal, so gut es ging - es fehlte das Verantwortungsbewußtsein gegenüber dem Ganzen. Ein solcher, in Iberoamerika tief verankerter Regionalismo steht auch heute noch einer zentralen Erfassung der Kräfte entgegen. - Zudem verlangt das Klima kaum die Vorsorge wie in nördlichen Breiten. Das wirtschaftliche Denken war bisher vielfach auf Improvisation und Spekulation eingestellt. So waren es auch mindestens bis in die fünfziger Jahre hinein überwiegend Ausländer, die langfristige Investierungen vornahmen. Die Industrialiserung erfordert eine Abkehr von der bisherigen Mentalität.

Mit dem Wunschdenken verbindet sich eine Neigung zum Formalismus. Allein schon durch Aufstellung eines Planes empfindet man eine Befriedigung, seine Realisierung tritt in der Bedeutung zurück ${ }^{20}$. Es deutet sich hier eine Parallelität zum Verfassungsbereich an, auf dem die zwanzig iberoamerikanischen Republiken seit rund 150 Jahren es bis 1965 bereits auf 186 Verfassungstexte gebracht haben ${ }^{21}$. Dabei ist die klaffende Lücke zwischen Verfassungstext und politischer Wirklichkeit nur allzu bekannt.

FRIEDRICH WEHNER

17 Joseph H. Kaiser, in: Planung I, S. 14 f., Nomos Verlag, Baden-Baden 1965.

18 Edward D. Beechert jr., The Gap between Planning Goals and Achievement in Latin America, S. 67, in: Inter-American Economic Affairs, Vol. 19, Nr. 1, 1965.

19 Frank Tannenbaum, Lateinamerika, S. 64 ff., W. Kohlhammer, Stuttgart 1963.

20 Beechert, a. a. O., S. $59 \mathrm{ff}$.

21 Mecham, a. a. O., S. 35. 